\title{
Lattice QCD results for the HVP contribution to the anomalous magnetic moments of leptons
}

\author{
Budapest-Marseille-Wuppertal Collaboration
}

Szabolcs Borsanyi ${ }^{1}$, Zoltan Fodor ${ }^{1,2,3}$, Taichi Kawanai ${ }^{3}$, Stefan Krieg ${ }^{1,3}$, Laurent Lellouch ${ }^{4}$, Rehan Malak $^{4,5}$, Kohtaroh Miura ${ }^{4, \star}$, Kalman K. Szabo ${ }^{1,3}$, Christian Torrero ${ }^{4}$, and Balint C. Toth ${ }^{1}$

${ }^{1}$ Department of Physics, Bergische Universität Wuppertal, Gaussstr. 20, D-42119 Wuppertal, Germany

${ }^{2}$ Inst. for Theor. Physics, Eötvös University, Pázmány P. sét. 1/A, H-1117 Budapest, Hungary

${ }^{3}$ Jülich Supercomputing Centre, Forschungszentrum Jülich, D-52425 Jülich, Germany

${ }^{4}$ Aix-Marseille U., U. de Toulon, CNRS, CPT, UMR 7332, F-13288 Marseille, France

${ }^{5}$ CNRS, CEA, Maison de la Simulation, USR 3441, F-91191 Gif-sur-Yvette Cedex, France

\begin{abstract}
We present lattice QCD results by the Budapest-Marseille-Wuppertal (BMW) Collaboration for the leading-order contribution of the hadron vacuum polarization (LOHVP) to the anomalous magnetic moments of all charged leptons. Calculations are performed with $\mathrm{u}, \mathrm{d}, \mathrm{s}$ and $\mathrm{c}$ quarks at their physical masses, in volumes of linear extent larger than $6 \mathrm{fm}$, and at six values of the lattice spacing, allowing for controlled continuum extrapolations. All connected and disconnected contributions are calculated for not only the muon but also the electron and tau anomalous magnetic moments. Systematic uncertainties are thoroughly discussed and comparisons with other calculations and phenomenological estimates are made.
\end{abstract}

\section{Introduction}

The muon's anomalous magnetic moments $a_{\mu}=\left(g_{\mu}-2\right) / 2$, with $g_{\mu}$ the Landé g-factor, is determined experimentally with a high precision of $0.5 \mathrm{ppm}$ [1], and forthcoming experiments aim to improve uncertainties to around $0.15 \mathrm{ppm}$ at Fermilab [2] and at J-PARC with similar objectives [3]. Comparisons of the experimental results with Standard Model (SM) predictions, accurate to about $0.4 \mathrm{ppm}$ [4], indicate a more than 3 standard deviation tension. This is in sharp contrast to the electron case $\left(a_{e}\right)$ in which the SM agrees to experiments in the ppb level. The tension in the muon case could be a sign of new, fundamental physics. Indeed, in many extensions of the SM, the new physics contributions are proportional to the lepton mass squared, and the corrections to $a_{\mu}$ would be roughly 40,000 times larger than those to $a_{e}$. The anomalous magnetic moments of $\tau$ lepton $\left(a_{\tau}\right)$ with the mass 17 times heaver than muon is even more sensitive for new physics, but the short lifetime of $\tau$ has made such a measurement impossible.

Theoretically, the leading source of uncertainty (over 78\% of the total) in the SM prediction of $a_{\mu}$ emerges from the leading order (LO) hadronic vacuum polarization (HVP) contribution, $a_{\mu}^{\mathrm{LO}-\mathrm{HVP}}$ [5]. The LO-HVP contribution also gives a dominant uncertainty in $a_{\tau}$ [6] and significant on the scale

${ }^{\star}$ Speaker, e-mail: kohtaroh.miura@cpt.univ-mrs.fr 


\begin{tabular}{cccccc}
\hline \hline$\beta$ & $a[\mathrm{fm}]$ & $\left(T \times L / a^{2}\right)$ & \#conf(uds/c/disc) & \#src(ud/s/c/disc) & bin-size(uds/c/disc) \\
\hline 3.7000 & 0.134 & $64 \times 48$ & $1000 / 40 / 1000$ & $768 / 64 / 4 / 9000$ & $10 / 1 / 10$ \\
3.7500 & 0.118 & $96 \times 56$ & $1500 / 40 / 1500$ & $768 / 64 / 4 / 6000$ & $10 / 1 / 10$ \\
3.7753 & 0.111 & $84 \times 56$ & $1500 / 40 / 1500$ & $768 / 64 / 4 / 6144$ & $10 / 1 / 10$ \\
3.8400 & 0.095 & $96 \times 64$ & $2500 / 40 / 1500$ & $768 / 64 / 4 / 3600$ & $10 / 1 / 10$ \\
3.9200 & 0.078 & $128 \times 80$ & $3500 / 40 / 1000$ & $768 / 64 / 4 / 6144$ & $10 / 1 / 10$ \\
4.0126 & 0.064 & $144 \times 96$ & $450 / 40 /-$ & $768 / 64 / 4 /-$ & $10 / 1 / 10$ \\
\hline \hline
\end{tabular}

Table 1. Parameters of the simulations performed. Each configurations are taken every 10 trajectories. The right-most column shows the configuration number in jackknife bin.

of the experimental error for $a_{e}$ [7]. Today $a_{\mu}^{\mathrm{LO}-\mathrm{HVP}}$ is determined most precisely using dispersion relations and the cross section of $e^{+} e^{-}$to hadrons and/or the rate of hadronic $\tau$ decays (see [5,8] and references therein). However, completely independent cross-checks by lattice QCD, without recourse to any experimental inputs, are important for testing the SM. Since the pioneering work of [9], lattice QCD calculations of $a_{\mu}^{\mathrm{LO}-\mathrm{HVP}}$ (see [10] and references therein) have made significant progress and will become competitive in the coming years.

In this proceedings, we present lattice QCD calculations of the $a_{\ell}^{\mathrm{LO}-\mathrm{HVP}}$ of all three leptons $(\ell=$ $e, \mu, \tau)[11]$ based on the same ensembles as used in [10]. We employ a tree-level improved Symanzik gauge action and a fermion action for four flavors of stout-smeared, rooted, staggered quarks. We have generated 15 ensembles at six values of the bare coupling, $\beta$, corresponding to lattice spacings ranging from 0.064 to $0.134 \mathrm{fm}$. We include all contributions from $u, d, s$ and $c$ quarks, in their quark-connected and quark-disconnected configurations. The up and down quark masses are taken to be degenerate and, together with the strange quark mass, they are tuned to around the physical mass point defined using the Goldstone pion and kaon masses. The charm quark mass is fixed in units of the strange mass to its physical value, $m_{c} / m_{s}=11.85$ [12]. The spatial (temporal) dimension of our lattices are in the range $6.1 \div 6.6(8.6 \div 11.3) \mathrm{fm}$. The lattice spacing is fixed with the pion leptonic decay constant, $f_{\pi}$. More information about the simulations can be found in [13].

In contrast to recent lattice works [14-16], our simulations are carried out at six values of the lattice spacing directly at the physical values of their masses, allowing for controlled continuum extrapolations. In particular, the continuum extrapolation of the disconnected contribution at the physical point is important for work related in the literature. Moreover, we implement a description of the lattice results $[17,18]$ that solves the small virtuality issue [19] with finite-volume (FV) artefacts that are exponentially suppressed in lattice size. The inclusion of all flavors up to the charm allows a controlled matching onto perturbation theory.

\section{Methodology}

We consider the two-point function of the quark electromagnetic current in euclidean time $t$ at zero spatial-momentum:

$$
C_{\mu \nu}(t)=\frac{1}{e^{2}} \int d^{3} x\left\langle j_{\mu}(x) j_{v}(0)\right\rangle,
$$

with $e$ the positron charge, $x=(t, \vec{x})$ and $j_{\mu} / e=\frac{2}{3} \bar{u} \gamma_{\mu} u-\frac{1}{3} \bar{d} \gamma_{\mu} d-\frac{1}{3} \bar{s} \gamma_{\mu} s+\frac{2}{3} \bar{c} \gamma_{\mu} c$. We work in the isospin limit, $m_{u}=m_{d}$. It is useful to split $C_{\mu \nu}$ into either flavor or isospin components since they have different statistical/systematic uncertainties:

$$
C_{\mu \nu}(t)=C_{\mu \nu}^{u d}(t)+C_{\mu \nu}^{s}(t)+C_{\mu \nu}^{c}(t)+C_{\mu \nu}^{\mathrm{disc}}(t)=C_{\mu \nu}^{I=1}(t)+C_{\mu \nu}^{I=0}(t)
$$


where in the first equality, the first three terms correspond to the quark-connected contractions of the light ( $u$ and $d$ combined), strange and charm quarks, and the fourth to the quark-disconnected contractions of $u, d$, and $s$. In the second equality, the separation is made between isospin $I=1$ and $I=0$ contributions, given by $C_{\mu \nu}^{I=1}=\frac{9}{10} C_{\mu \nu}^{u d}$ and $C_{\mu \nu}^{I=0}=\frac{1}{10} C_{\mu \nu}^{u d}+C_{\mu \nu}^{s}+C_{\mu \nu}^{c}+C_{\mu \nu}^{\text {disc }}$.

The LO-HVP contributions to the anomalous magnetic moment of lepton $\ell$ are obtained from those of scalar HVP functions as [9, 20]

$$
a_{\ell, f}^{\mathrm{LO}-\mathrm{HVP}}=\left(\frac{\alpha}{\pi}\right)^{2} \int_{0}^{\infty} \frac{d Q^{2}}{m_{\ell}^{2}} \omega\left(\frac{Q^{2}}{m_{\ell}^{2}}\right) \hat{\Pi}^{f}\left(Q^{2}\right),
$$

with $\alpha=e^{2} /(4 \pi), \omega(r)=\pi^{2}[r+2-\sqrt{r(r+4)}]^{2} / \sqrt{r(r+4)}$, and the $\hat{\Pi}^{f}\left(Q^{2}\right)$ is given by [17]

$$
\hat{\Pi}^{f}\left(Q^{2}\right) \equiv \Pi^{f}\left(Q^{2}\right)-\Pi^{f}(0)=\frac{1}{3} \sum_{i=1}^{3} \int_{0}^{\infty} d t\left[t^{2}-\frac{4}{Q^{2}} \sin ^{2}\left(\frac{Q t}{2}\right)\right] C_{i i}^{f}(t) .
$$

In Eqs. (3) and (4), $f=u d, s, c$, disc, $I=1, I=0, \sqcup$ and the " $\sqcup$ " indicates that this equation also applies to the full LO-HVP contribution. One can construct the $\Pi^{f}\left(Q^{2}\right)$ in terms of the hypercubic transformations of $C_{i i}^{f}(t)$ in Eq. (4), but they do not increase our overall accuracy, and are not taken account.

On a $T \times L^{3}$ lattice with spacing $a$, the integral over $t$ in Eq. (4) is replaced by a sum, in increments of $a$, that runs up to $T / 2$, once the correlator $C_{i i}^{f}(t)$ has been appropriately averaged with $C_{i i}^{f}(T-t)$. Moreover, the integral over $Q$ in Eq. (4) should, in principle, be replaced by a sum from 0 to $\pi / a$ in steps of $2 \pi / T$, but we here keep the integral; it should be noted, however, we need to introduce an upper bound $Q=Q_{\max }$ in the integral. It is chosen much smaller than $\pi / a$, such that discretization errors are under controlled, but perturbation theory can be applied for $Q>Q_{\max }$ :

$$
a_{\ell, f}^{\mathrm{LO}-\mathrm{HVP}}=a_{\ell, f}^{\mathrm{LO}-\mathrm{HVP}}\left(Q \leq Q_{\max }\right)+\gamma_{\ell}\left(Q_{\max }\right) \hat{\Pi}^{f}\left(Q_{\max }^{2}\right)+\Delta^{\mathrm{pert}} a_{\ell, f}^{\mathrm{LO}-\mathrm{HVP}}\left(Q>Q_{\max }\right) .
$$

Here, the low momentum contribution, $a_{\ell, f}^{\mathrm{LO}-\mathrm{HVP}}\left(Q \leq Q_{\max }\right)$, is obtained from the lattice measurements for the correlator Eq. (1). We use the conserved lattice current at the source and sink so that no renormalization is necessary. See Table 1 for details on the simulation setup. The last term in Eq. (5) is the high-momentum, perturbative contribution renormalized at $Q_{\max }$. The second term is required to shift the renormalization point from $Q_{\max }$ to $Q=0$. It is obtained with lattice results for $C_{i i}^{f}(t)$, through Eq. (4) with $Q=Q_{\max }$. The $\gamma_{\ell}\left(Q_{\max }\right)$ is a known kinematical factor: $\gamma_{\ell}\left(Q_{\max }^{2}\right)=\left(\frac{\alpha}{\pi}\right)^{2} \int_{Q_{\max }}^{\infty} \frac{d Q}{m_{\ell}} \omega\left(Q^{2} / m_{\ell}^{2}\right)$, where the $\omega(r)$ is defined after Eq. (3). In obtaining Eq. (5), it is assumed that $\Delta^{\text {pert }} a_{\ell, f}^{\mathrm{LO}-\mathrm{HVP}}\left(Q>Q_{\max }\right)$ is equal to the value that it would have nonperturbatively. We check this by studying the dependence of our results on the choice of $Q_{\max }$.

The light and disconnected correlators $\left(C_{i i}^{u d}(t)\right.$ and $\left.C_{i i}^{\text {disc }}(t)\right)$, are notoriously noisy at large distance. This is true even with our high statistics. To address this problem, we introduce a cut $t_{c}$ fm in time in Eq. (4), and replace the $C_{i i}^{u d}(t)\left(-C_{i i}^{\text {disc }}(t)\right)$ by an upper and a lower bound for $t>t_{c}$. Both $C_{i i}^{u d}(t)$ and $-C_{i i}^{\text {disc }}(t)$ are strictly positive and the lower bounds are given by zero. For the upper bounds, we adopt $C_{i i}^{u d}\left(t_{c}\right) \frac{\varphi(t)}{\varphi\left(t_{c}\right)}$ for the light component and $(1 / 10) C_{i i}^{u d}\left(t_{c}\right) \frac{\varphi(t)}{\varphi\left(t_{c}\right)}$ for the minus of the disconnected components. Here, $\varphi(t)=\cosh \left[E_{2 \pi}(T / 2-t)\right]$ with $E_{2 \pi}$ denoting energy of two pions. Thus, the light/disconnected contributions to HVP are obtained by summing the integrand in Eq. (4) with $C_{i i}^{u d / \text { disc }}(t)$ given by our lattice data up to $t_{c}$ and performing the rest of the sum from $t>t_{c}$ to $T / 2$ with $C_{i i}^{u d / \text { disc }}(t)$ replaced by the average discussed above.

The $t_{c}$ is chosen as such that the upper and lower bounds agree well within statistical errors and where these errors are not too large [10]. For light components, this condition is satisfied around 3.0 
$\mathrm{fm}$. We consider various $t_{c}$ in the range of $(3.000 \pm 0.134) \mathrm{fm}$, and adopt the average of $a_{\ell}^{\mathrm{LO}-\mathrm{HVP}}$ over $t_{c}$ as a value to be used in later. The systematic error is given by fluctuations over $t_{c}$. The same procedure is carried out for the disconnected part with $t_{c}$ in the range of $(2.600 \pm 0.134) \mathrm{fm}$.

\section{Results}

\subsection{Continuum Extrapolation}

The continuum extrapolations are performed for $a_{\ell, f}^{\mathrm{LO}-\mathrm{HVP}}\left(Q \leq Q_{\max }\right)$, the first term of the right-hand side in Eq. (5), for which we use our lattice data. Hereafter, quantities with the subscript "lat" correspond to lattice results obtained in a given simulation: $a_{\ell, f, \text { lat }}^{\mathrm{LO}}\left(Q \leq Q_{\max }\right)$. To extrapolate our results $a_{\ell, f \text { lat }}^{\mathrm{LO}-\mathrm{HVP}}\left(Q \leq Q_{\max }\right)$ to the continuum limit and to interpolate them to the physical mass point, we fit them to a function which depends on the lattice spacing squared with or without the Goldstone pion/kaon masses squared and/or $\eta_{c}$ meson mass (not squared).

We adopt a linear ansatz in $a^{2}$. To take account of systematics associated with discretizations, we impose four cuts on the lattice spacing (no cut, and $a \leq 0.118,0.111$, $0.095 \mathrm{fm}$ ) in the continuum extrapolation. This number is reduced to three in the disconnected case for which we have no results at $a=0.064 \mathrm{fm}$. Since the simulations are performed close to the physical mass point, a constant or linear pion/kaon mass-squared or $\eta_{c}$ mass dependence is always sufficient. For the light-quark and disconnected contributions, the dependence on meson masses is not significant statistically and can safely be ignored.

In Fig. 1, we show our results for the muon case obtained with $Q_{\max }=2 \mathrm{GeV}$ for all flavors, $a_{\mu, f \text {, lat }}^{\mathrm{LO}}(Q \leq 2 \mathrm{GeV})(f=u d, s, c$, disc $)$ as a function of $a^{2}$. As can be seen in

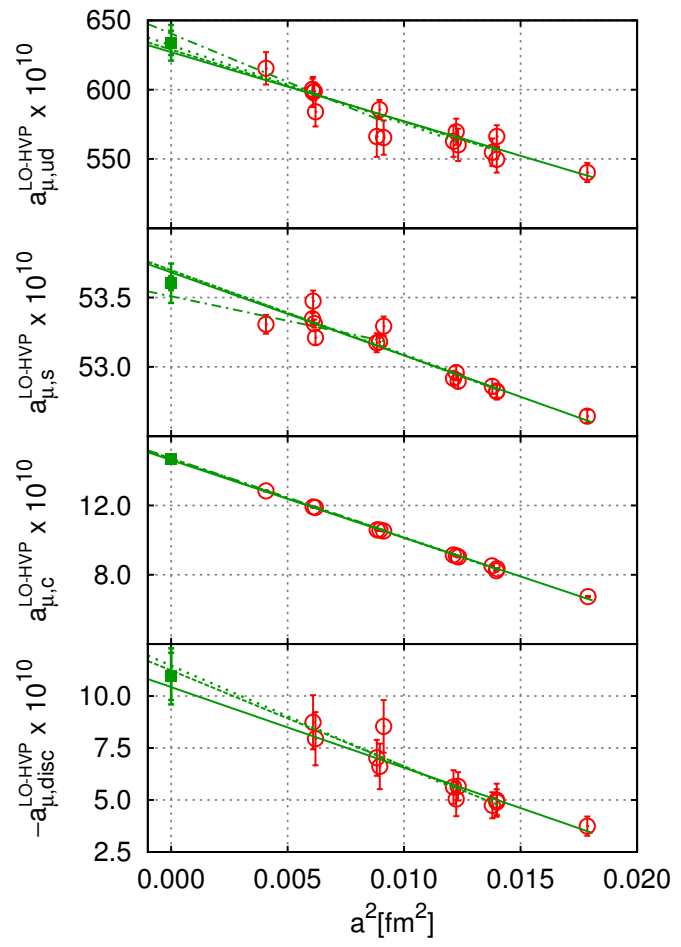

Figure 1. Continuum extrapolation of the various flavor contributions to $a_{\mu}^{\mathrm{LO}-\mathrm{HVP}}(Q \leq 2 \mathrm{GeV})$, plotted as a function of lattice spacing squared. See text for details. The green squares at $a^{2}=0$ are our continuum limits with statistical and systematic errors. the top panel of Fig. 1, the light component $a_{\mu, u d, \text { lat }}^{\mathrm{LO}}(Q \leq 2 \mathrm{GeV})$ shows a strong $a^{2}$ dependence: the coarsest lattice is about $16 \%$ smaller than the continuum limit. This is due to the sensitivity of this contribution to low-energy, two-pion states which, in turn, are sensitive to taste splittings. For the strange contribution $a_{\mu, s, \text { lat }}^{\mathrm{LO}}(Q \leq 2 \mathrm{GeV})$, the kaon mass correction term $\left(M_{K x}^{2} \equiv M_{K}^{2}-M_{\pi}^{2} / 2\right)$ is needed to get a reasonable fit quality. The taste violations is much smaller than the light component case as seen in the second panel of Fig. 1.

In advance to the analyses for the charm contribution $a_{\mu, c, \text { lat }}^{\mathrm{LO}}(Q \leq 2 \mathrm{GeV})$, we studied the continuum extrapolation of $D_{s}$ meson mass $\left(M_{D s}\right)$, and found that $M_{\eta c}$ correction term is necessary in 
the fit model to reproduce the physical value $M_{D s}^{\text {phys }}$ [21]; via fixing $M_{\eta c}$ to its physical value, a little mistuning effect $(\sim 2 \%)$ of the ratio $m_{c} / m_{s}$ is corrected. We also apply this correction to $a_{\mu, c}^{\mathrm{LO}-\mathrm{HVP}}$ extrapolation. In addition, we include a pion mass $M_{\pi}^{2}$ correction but not the kaon mass term, where the latter results in overfitting due to the fixed $m_{c} / m_{s}$. The dependence on $a^{2}$ is strong; as shown in the third panel of Fig. 1, our coarsest lattice is more than 50\% smaller than the continuum limit, due to the large value of $m_{c}$ in lattice units. To get reasonable fit qualities for charm contributions, we consider the subset of 40 configurations most separated in molecular dynamics time, which provides more than enough statistics for this contribution.

Our results for $a_{\mu \text {,disc,lat }}^{\mathrm{LO}}(Q \leq 2 \mathrm{GeV})$ show the strong lattice artefacts, as shown in the bottom panel of Fig. 1. These are also due to the taste violations. The disconnected part includes both flavordiagonal and off-diagonal contributions, in which we take account of $u d$ and $s$ but neglect $c$; the charm contribution amounts to less than $1 \%$ of the total disconnected contribution on our coarsest lattice and much smaller than the statistical error. In addition, because statistical errors are quite large, no dependence on quark mass is required to describe the lattice data.

We repeat this whole analysis for $Q_{\max }=1, \sqrt{2}, \sqrt{3}$ and $\sqrt{5} \mathrm{GeV}$ in $a_{\ell, f, \text { lat }}^{\mathrm{LO}-\mathrm{HVP}}\left(Q \leq Q_{\max }\right)$ and in $\hat{\Pi}^{f}\left(Q_{\max }^{2}\right)$ using Eq. (4), where the latter is necessary for the second term in Eq. (5).

\subsection{Matching to Perturbation}

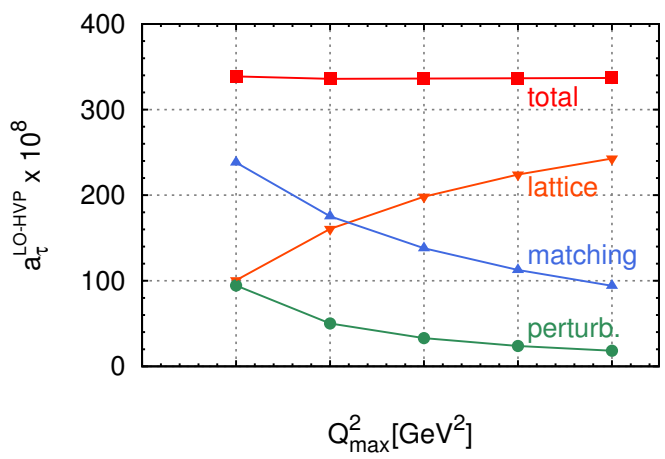

Figure 2. The $Q=Q_{\max }$ dependence of $a_{\tau}^{\mathrm{LO}-\mathrm{HVP}}$. The upside-down orange triangles, blue triangles and green circles correspond to the first, second, and third term in Eq. (5), respectively. The red squares represent sum of the three. Error bars are smaller than the symbols.

So far, we have focused on the low momentum contribution (first term in Eq. (5)); we now move onto the other contributions (the second and third therms in Eq. (5)). They are computed from our lattice results for $\Pi^{f}\left(Q^{2}\right)$ (second term) and five-loop perturbation theory (third term) with the code rhad [22]. These corrections are below our statistical errors for the $e$ and $\mu$, which have very little sensitivity to large $Q$, but are significant for the $\tau$. As shown in Fig. 2, however, the $Q_{\max }$ dependence of high momentum effects are almost canceled by those in the low momentum of our lattice results, and the total (red squares) are stable against $Q_{\max }$. Thus, the continuum limits of our lattice data $a_{\ell, f, \text { lat }}^{\mathrm{LO}}\left(Q \leq Q_{\max }\right)$ are consistent with five-loop perturbation theory for momenta, $Q>Q_{\max }$. More quantitatively, a weak $Q_{\max }$ dependence beyond statistical errors starts appearing for $Q<\sqrt{2} \mathrm{GeV}$, indicating a break down of perturbation theory, and we adopt the safe region of $\sqrt{2} \leq Q_{\max } \leq \sqrt{5}$ $\mathrm{GeV}$ for our final result.

The whole procedure described above gives us $a_{\ell, f}^{\mathrm{LO}-\mathrm{HVP}}$ for $f=u d, s, c$, disc, $I=1, I=0,\llcorner$ and for all three leptons. As explained above, there are three sources of systematic errors: a-cut $=$ the data cutting in continuum extrapolations, $\mathrm{t}-\mathrm{cut}=$ the cuts associated with the large temporal distance treatment $t_{c}$, 
Q-cut $=$ the high/low momentum separation $Q_{\max }$. The final central value is taken to be the mean in the flat distribution over the variations of the above three cuts [23, 24]. The statistical error is the jackknife error of the central value over jackknife samples with bins of length 10 configurations (for charm, 1 configuration). For each of a-cut, t-cut, and Q-cut, the associated systematic error is the maximum deviation from the central value. In addition, we put the systematic error associated with the uncertainty in the lattice spacing determination by $0.4 \%$, which amounts to $0.8 \%$ uncertainty on $a_{\ell, f}^{\mathrm{LO}-\mathrm{HVP}}$. The total systematic error is obtained by adding all systematic errors in quadrature.

\subsubsection{FV, Isospin Breaking, and QED Corrections}

The results obtained above are further corrected in terms of finite volume, isospin breaking, and QED effects. In the absence of a systematic study with simulations in a variety of volumes, only model estimates of FV effects can be made. As argued in [25, 26], for large volumes those effects will be governed by pion contributions that can be computed in chiral perturbation theory $(\chi \mathrm{PT})$ [25]. Since the $I=0$ channel is dominated by three-pion exchange, the FV effects are expected to be smaller than those of the $I=1$ contribution, which are already small. Thus we consider only the latter, and the appropriate corrections are added to our $I=1$ and total results. We associate with these corrections a $100 \%$ uncertainty included in our error budget.

Compared to phenomenological determinations of $a_{\mu}^{\mathrm{LO}-\mathrm{HVP}},[5,8]$ our $m_{d}=m_{u}$ calculation without QED is missing a number of effects. We correct those effects as follows: the $\pi^{0} \gamma$ and $\eta \gamma$ contributions for which can be taken directly from the dispersive approach [27], final-state radiation (FSR) for which we use the results from [27] and attribute to it a 50\% error, the $\rho$ - $\omega$ mixing for which we take from [27] and conservatively attribute to it a 50\% error, the QED mass shift of charged pions by LO in $\chi \mathrm{PT}$ using $M_{\pi^{ \pm}}$and $M_{\pi}$ with $100 \%$ uncertainty to cover other, neglected effects.

\section{Summary and Discussion}

Putting everything together, we obtain our final results for all three leptons [11]: $a_{\mu}^{\mathrm{LO}-\mathrm{HVP}}=$ $713(9)(18), a_{e}^{\mathrm{LO}-\mathrm{HVP}}=189(3)(6), a_{\tau}^{\mathrm{LO}-\mathrm{HVP}}=342(1)(4)$, where the first and second brackets are the statistical and systematic uncertainties, respectively. In the Fig. 3, we compare our final result of $a_{\mu}^{\mathrm{LO}-\mathrm{HVP}}$ with those in lattice QCD determinations including the contributions of quarks up to the charm [14, 28] and recent phenomenological estimates [5, 8]. We also compare with the value that $a_{\mu}^{\mathrm{LO}-\mathrm{HVP}}$ would have to explain the experimental measurement of $a_{\mu}$ [1] within SM, i.e. assuming no new physics. This amounts to $a_{\mu, \text { noNP }}^{\mathrm{LO}}=(720.0 \pm 6.8) \times 10^{-10}$ by taking account of all SM contributions summarized in [5], and shown by the light-blue band in the figure. Our result for $a_{\mu}^{\mathrm{LO}-\mathrm{HVP}}$ is larger than those of the other lattice calculations. In particular, our $a_{\mu}^{\mathrm{LO}-\mathrm{HVP}}$ shows about $2.0 \sigma$ tension to the HPQCD result [28]. This namely originates from the larger value of the light component contribution in our result. However, our $a_{\mu}^{\mathrm{LO}-\mathrm{HVP}}$ is consistent with both phenomenological estimates with dispersion relations and the $a_{\mu, \text { noNP }}^{\mathrm{LO}}$ within one and some standard deviations.

For $a_{e, \tau}^{\mathrm{LO}-\mathrm{HVP}}$, we find two lattice calculations $[16,28]$ and phenomenological ones [6, 7]. Our $a_{e}^{\mathrm{LO}-\mathrm{HVP}}$ is somewhat larger but consistent to the estimate in [16] within the uncertainty, while there exists a tension to the result in [28]. It is remarkable that our results are fully compatible with the phenomenological ones. This suggests that the physics of the HVP over full range of $Q^{2}$ is wellcontrolled. Our results for both $a_{e}^{\mathrm{LO}-\mathrm{HVP}}$ and $a_{\tau}^{\mathrm{LO}-\mathrm{HVP}}$ are determined with much smaller uncertainties than those of [16]. For $a_{\tau}^{\mathrm{LO}-\mathrm{HVP}}$, our result is more precise even comparing to the phenomenological estimates [6]. 


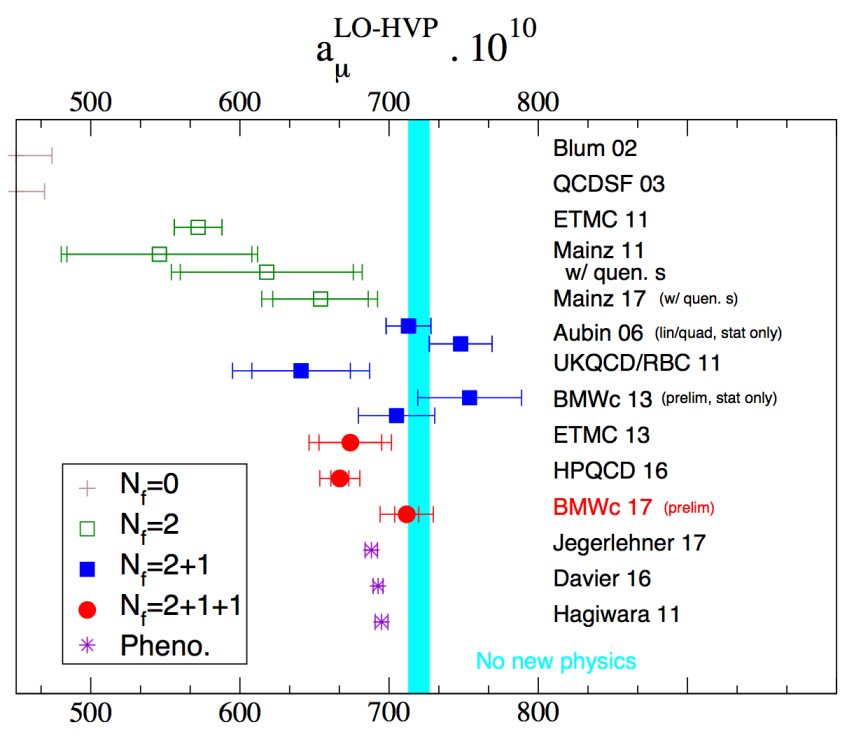

Figure 3. Comparisons of our result for $a_{\mu}^{\mathrm{LO}-\mathrm{HVP}}$ with recently reported ones in lattice QCD [14, 28], with ones in phenomenology $[5,8]$, and with the value which have to explain the experimental measurement of $a_{\mu}$, assuming no new physics (light-blue band). For the lattice results, the first error is statistical and the second is the total error, including systematics.

The total error (statistical and all systematic uncertainties added in quadrature) in our $a_{\mu}^{\mathrm{LO}-\mathrm{HVP}}$ is a few percent level. In order to confirm or infirm the tension between the measurement of $a_{\mu}$ and the prediction of the SM based on phenomenology, we need to decrease a total uncertainty. To this end, we plan to increase statistics, control finite volume effects directly with simulations, and perform simulations with QED and isospin breaking effects.

\section{Acknowledgments}

LL thanks M. Benayoun, C. Davies, F. Jegerlehner, C. Lehner, E. de Rafael and R. Van de Water for very informative discussions. Computations were performed on JUQUEEN and JUROPA at Forschungszentrum Jülich, on Turing at the Institute for Development and Resources in Intensive Scientific Computing (IDRIS) in Orsay, on SuperMUC at Leibniz Supercomputing Centre in München, on Hermit at the High Performance Computing Center in Stuttgart. This project was supported by in part by the OCEVU Laboratoire d'excellence (ANR-11-LABX-0060) and the A*MIDEX Project (ANR-11-IDEX-0001-02), which are funded by the "Investissements d'avenir" French government program and managed by the "Agence nationale de la recherche" (ANR), by the DFG Grant SFB/TR55, by the Gauss Centre for Supercomputing e.V and by the GENCI-IDRIS supercomputing grant No. 52275. RM was supported in part by a joint Ph.D. fellowship from the Centre national de la recherche scientifique (CNRS) and the Commissariat à l'énergie atomique et aux énergies alternatives (CEA).

\section{References}

[1] G.W. Bennett et al. (Muon g-2), Phys. Rev. D73, 072003 (2006), hep-ex/0602035

[2] J.L. Holzbauer, J. Phys. Conf. Ser. 770, 012038 (2016), 1610. 10069

[3] M. Otani (E34), JPS Conf. Proc. 8, 025008 (2015)

[4] T. Blum, A. Denig, I. Logashenko, E. de Rafael, B. Lee Roberts, T. Teubner, G. Venanzoni (2013), 1311.2198 
[5] M. Davier, Update of the Hadronic Vacuum Polarisation Contribution to the muon g-2, in 14th International Workshop on Tau Lepton Physics (TAU 2016) Beijing, China, September 19-23, 2016 (2016), 1612.02743

[6] S. Eidelman, M. Passera, Mod. Phys. Lett. A22, 159 (2007), hep-ph/0701260

[7] F. Jegerlehner, EPJ Web Conf. 118, 01016 (2016), 1511.04473

[8] F. Jegerlehner, Muon g-2 Theory: the Hadronic Part, in KLOE-2 workshop on $e^{+} e^{-}$collider physics at $1 \mathrm{GeV}$, INFN-Laboratori Nazionali di Frascati, Italy, 26-28 October 2016 (2017), 1705.00263

[9] T. Blum, Phys. Rev. Lett. 91, 052001 (2003), hep-lat/0212018

[10] S. Borsanyi, Z. Fodor, T. Kawanai, S. Krieg, L. Lellouch, R. Malak, K. Miura, K.K. Szabo, C. Torrero, B. Toth (Budapest-Marseille-Wuppertal) (2016), 1612 .02364v1

[11] S. Borsanyi, Z. Fodor, T. Kawanai, S. Krieg, L. Lellouch, R. Malak, K. Miura, K.K. Szabo, C. Torrero, B. Toth (Budapest-Marseille-Wuppertal) (In Preparation)

[12] C.T.H. Davies, C. McNeile, K.Y. Wong, E. Follana, R. Horgan, K. Hornbostel, G.P. Lepage, J. Shigemitsu, H. Trottier, Phys. Rev. Lett. 104, 132003 (2010), 0910. 3102

[13] R. Bellwied, S. Borsanyi, Z. Fodor, S.D. Katz, A. Pasztor, C. Ratti, K.K. Szabo, Phys. Rev. D92, 114505 (2015), 1507.04627

[14] F. Burger et al. (ETM), JHEP 1402, 099 (2014), 1308. 4327

[15] B. Chakraborty, C.T.H. Davies, J. Koponen, G.P. Lepage, M.J. Peardon, S.M. Ryan, Phys. Rev. D93, 074509 (2016), 1512.03270

[16] F. Burger, K. Jansen, M. Petschlies, G. Pientka, Eur. Phys. J. C76, 464 (2016), 1501.05110

[17] D. Bernecker, H.B. Meyer, Eur.Phys.J. A47, 148 (2011), 1107.4388

[18] M. Spraggs, P. Boyle, L. Del Debbio, A. Jüttner, C. Lehner, K. Maltman, M. Marinkovic, A. Portelli, PoS LATTICE2015, 106 (2016), 1601.00537

[19] C. Aubin, T. Blum, M. Golterman, S. Peris, Phys. Rev. D86, 054509 (2012), 1205 . 3695

[20] B.E. Lautrup, E. De Rafael, Phys. Rev. 174, 1835 (1968)

[21] K. Cichy, M. Kalinowski, M. Wagner, Phys. Rev. D94, 094503 (2016), 1603.06467

[22] R.V. Harlander, M. Steinhauser, Comput. Phys. Commun. 153, 244 (2003), hep-ph/0212294

[23] S. Borsanyi et al. (Budapest-Marseille-Wuppertal), Science 347, 1452 (2015), 1406.4088

[24] S. Durr et al. (Budapest-Marseille-Wuppertal), Science 322, 1224 (2008), 0906.3599

[25] C. Aubin, T. Blum, P. Chau, M. Golterman, S. Peris, C. Tu, Phys. Rev. D93, 054508 (2015), 1512.07555

[26] A. Francis, B. Jaeger, H.B. Meyer, H. Wittig, Phys.Rev. D88, 054502 (2013), 1306.2532

[27] F. Jegerlehner, private communication, June 2017.

[28] B. Chakraborty, C.T.H. Davies, P.G. de Oliviera, J. Koponen, G.P. Lepage, R.S. Van de Water, Phys. Rev. D96, 034516 (2017), 1601.03071 